\title{
Comparison of Dysphagia Between Infratentorial and Supratentorial Stroke Patients
}

\author{
Yong Kyun Kim, MD, PhD, Jung Hyun Cha, MD, Kyun Yeon Lee, MD
}

Department of Physical Medicine and Rehabilitation, Myongji Hospital, Goyang, Korea

\begin{abstract}
Objective To compare dysphagia between infratentorial stroke patients and supratentorial stroke patients. Methods Subjects of this study were patients with post-stroke dysphagia (PSD) who were admitted to our medical institution between May 2014 and June 2017. We evaluated a total of 64 patients with PSD. A videofluoroscopic swallowing study (VFSS) was performed to determine dysphagia severity. We measured the following parameters: pharyngeal transit time (PTT), post-swallow pharyngeal remnant, Penetration Aspiration Scale (PAS) scores, and Functional Dysphagia Scale (FDS). We analyzed patient's results from VFSS performed at admission. All VFSS images were recorded using a camcorder running at 30 frames per second. An AutoCAD 2D screen was used to measure post-swallow pharyngeal remnant.

Results In this study, PTT and FDS were similar $(\mathrm{p}>0.05)$ between infratentorial stroke patients and supratentorial stroke patients. However, there were significant differences in pharyngeal remnant and PAS scores between the two groups ( $\mathrm{p}<0.01$ and $\mathrm{p}<0.05$, respectively).

Conclusion Both pharyngeal remnant and PAS score registered higher levels from VFSS test for infratentorial stroke patients than those for supratentorial stroke patients. This suggests greater chances of problems occurring with swallowing, the major functions of pons. Thus, clinicians should pay particular attention to active dysphagia evaluation and treatment in PSD of infratentorial stroke patients.
\end{abstract}

Keywords Deglutition disorders, Stroke, Infratentorial, Supratentorial, Fluoroscopy

\section{INTRODUCTION}

Post-stroke dysphagia (PSD) is a common complication after a stroke, with a prevalence of $19 \%-81 \%[1,2]$. A specific relationship between the anatomical structure of the brain and the process of swallowing has not yet been identified. However, the swallowing center which is named the central pattern generator is generally known to be located in the medulla [3-5]. Patients with PSD are known to be at increased risk of pulmonary complications and mortality $[6,7]$. Therefore, it would be critical to perform accurate estimation of the incidence of PSD and

Received August 7, 2018; Accepted October 12, 2018

Corresponding author: Kyun Yeon Lee

Department of Physical Medicine and Rehabilitation, Myongji Hospital, 55 Hwasu-ro 14beon-gil, Deokyang-gu, Goyang 10475, Korea. Tel: +82-31-8106450, Fax: +82-31-810-5259, E-mail: uraneky@gmail.com

ORCID: Yong Kyun Kim (http://orcid.org/0000-0002-1224-8965); Jung Hyun Cha (http://orcid.org/0000-0002-7120-1091); Kyun Yeon Lee (http://orcid. org/0000-0001-8822-4006).

(c) This is an open-access article distributed under the terms of the Creative Commons Attribution Non-Commercial License (http://creativecommons.org/ licenses/by-nc/4.0) which permits unrestricted noncommercial use, distribution, and reproduction in any medium, provided the original work is properly cited. Copyright ๑ 2019 by Korean Academy of Rehabilitation Medicine 
risk of pulmonary complications in patients with stroke.

Because of these complications, previous studies have attempted to identify differences in swallowing function according to the location of the brain lesion in patients with PSD for establishing appropriate management [8]. A few previous studies have investigated swallowing function in patients with brainstem lesions without differentiating lesions and reported varying symptoms related to swallowing, including pharyngeal remnant, vocal cord paralysis, dysarthria, change in voice, vallecular fossa, pyriform sinus residue, pharyngeal transit time (PTT), and larynx elevation $[4,9,10]$. In one study, dysphagia developed more frequently in patients with a medial medullary infarction than that in those with lateral medullary infarction [5].

The objective of this study was to compare dysphagia between infratentorial stroke patients and supratentorial stroke patients. The severity of dysphagia was determined according to various locations associated with PSD in patients with a brainstem lesion including the swallowing center. In this study, patients were classified into infratentorial stroke group and supratentorial stroke group. All patients underwent videofluoroscopic swallowing study (VFSS).

\section{MATERIALS AND METHODS}

\section{Subjects}

This study included 64 patients with stroke admitted to the rehabilitation medicine department of Myongji Hospital from May 1, 2014 to June 30, 2017. This study was approved by the Institutional Review Board of our medical institution (No. 07-011). Inclusion criteria were (1) patients with stroke due to cerebral hemorrhage and cerebral infarction; (2) patients who underwent VFSS evaluation for the stroke indicated in (1); (3) patients aged over 18 years; and (4) patients who were on tube feeding at the time of performing VFSS test for PSD. Exclusion criteria were (1) patients with structural abnormalities of the oropharynx; (2) patients with structural abnormalities of the cervical spine (for example, patients with bony abnormalities like osteophytes); (3) patients with high possibility of broad brain injury such as massive intracranial hemorrhage (ICH), hypoxic brain injury, traumatic brain injury, or multiple infarction; and (4) patients with underlying neurologic disease (for example, Parkinson disease, dementia, or motor neuron disease).

\section{Measures}

VFSS results and medical charts of included patients were analyzed to assess the following parameters during semisolid swallowing: PTT, post-swallow pharyngeal remnant, Penetration Aspiration Scale (PAS) score, and Functional Dysphagia Scale (FDS) score.

We used the following definitions as previously described [11]. PTT (in ms) was defined as the period of time that a bolus remained in the pharynx, during which food passed from the pharyngeal fornix into the upper esophageal sphincter (UES). The delay in PTT was measured as the time required for the food bolus to pass the cricopharyngeal level from the lower corner of the mandible [12]. Post-swallow pharyngeal remnant (\%) was defined as the amount of food retained in the pharyngeal space. Its width corresponded to the area calculated based on the reference using 2D view in AutoCAD (Autodesk Inc., San Rafael, CA, USA). The reference length was a 100 -won coin ( $23 \mathrm{~mm}$ in diameter) attached to the neck [13]. PAS score (point) was an 8-point multidimensional indicator of airway invasion. The presence of airway penetration (entry of contrast material into the laryngeal vestibule) and aspiration (that below the level of the true vocal folds) were recorded for each swallow using PAS score [14]. To quantify VFSS test results, FDS (point) devised by Han et al. [4] was used. Lip closure, food bolus formation, and masticatory function in the oral phase were assessed against three levels. The residue inside the oral cavity after swallowing was assessed against four levels. The transit time for what was thought to be the main food bolus during swallowing was measured. During the pharyngeal phase, laryngeal elevation, decrease in epiglottic closing function, delay in swallowing reflex, and barium coating on the pharyngeal wall after swallowing were evaluated. Moreover, the amount of food bolus invasion into the nasal cavity, the amount of residue in the pyriform sinuses after swallowing, and the amount of residue in the vallecular fossa after swallowing were evaluated for classification to one of four levels.

\section{Procedure}

The VFSS test was used to determine whether objective examination indices such as aspiration, spatial, and temporal movements of the pharyngeal stage, pharyngeal 
remnant presence, and clinical severity were different depending on the location of the stroke. If so, the difference in severity was assessed. VFSS was performed using a semisolid $4 \mathrm{~mL}$ bolus in patients. At this hospital, two substances, including a semisolid and a liquid, were tested during the VFSS. For this study, we utilized a semisolid value that could be used to calculate the remnant amount. Patients who showed decreased cognition or difficulty in maintaining head control during the VFSS were seated in wheelchairs and an assistant helped them maintain a chin-tuck posture by holding the head [11]. All VFSS procedures were recorded using a camcorder at 30 frames per second. All images were saved in a personal computer and analyzed using a multimedia player (Gomplayer; Gretech, Seoul, Korea).

\section{Statistical analysis}

Data are expressed as mean \pm standard deviation. Statistical analysis was done using the SPSS version 18.0 for Windows (SPSS Inc., Chicago, IL, USA). We compared differences between the two groups using independent samples t-test. A p-value less than 0.05 was considered statistically significant.

\section{RESULTS}

In this study, we included a total of 64 patients with stroke admitted to the rehabilitation medicine department of our hospital from May 1, 2014 to June 30, 2017.
These patients were assigned to the infratentorial stroke group $(n=34)$ or the supratentorial group $(n=34)$. There was no statistically significant difference in general characteristics between the two groups (Table 1).

Although a significant difference was found with respect to pharyngeal remnant $(\mathrm{p}<0.01)$ and PAS score $(\mathrm{p}<0.05)$ between the two groups, there was no statistically significant difference in PTT ( $p>0.05)$ or FDS score ( $p>0.05)$ between the two groups (Fig. 1). Although the pharyngeal remnant was measured to be $20.69 \% \pm 15.23 \%$ in the supratentorial group, it was $40.92 \% \pm 17.12 \%$ in the pontine stroke group, showing nearly a two-fold difference in the thickness of remnant. The PAS score indicating the severity of dysphagia was $4.21 \pm 1.4$ in the supratentorial stroke group and $5.12 \pm 1.2$ in the infratentorial stroke group, showing a significant difference $(\mathrm{p}<0.05)$.

\section{DISCUSSION}

Dysphagia is a common complication that can develop after stroke [15]. If the stroke involves the area of the brainstem, even a small lesion can lead to a critical situation [10]. This is because the cranial nerve nuclei, nerve tract, and the reticular interneurons within the brainstem structures are clustered [9]. In one study, the prevalence of aspiration pneumonia in the infratentorial stroke group was nearly twice as high that in the supratentorial stroke group. It can be assumed that the risk of aspiration is higher in the infratentorial stroke group, although

Table 1. General characteristics of patients

\begin{tabular}{|lccc}
\hline \multicolumn{1}{|c}{ Characteristic } & $\begin{array}{c}\text { Infratentorial stroke } \\
\text { group }(\mathbf{n}=\mathbf{3 4})\end{array}$ & $\begin{array}{c}\text { Supratentorial stroke } \\
\text { group }(\mathbf{n}=\mathbf{3 4})\end{array}$ & p-value \\
\hline Age (yr) & $59.79 \pm 23.5$ & $60.4 \pm 22.3$ & 0.72 \\
\hline Sex & & & 0.63 \\
\hline Male & 21 & 17 & \\
\hline Female & 13 & 17 & 0.72 \\
\hline K-MMSE & $15.02 \pm 8.25$ & $11.85 \pm 11.45$ & 0.49 \\
\hline Duration of disease (mo) & $45.54 \pm 12.65$ & $41.12 \pm 17.78$ & 0.99 \\
\hline Stroke type & 16 & & 0.86 \\
\hline Ischemic & 18 & 15 & 0.82 \\
\hline Hemorrhagic & $17.23 \pm 5.21$ & 19 & 0.11 \\
\hline NIHSS & 29 & $13.75 \pm 7.94$ & \\
\hline Aspiration pneumonia history & & & \\
\hline
\end{tabular}

Values are presented as mean \pm standard deviation.

K-MMSE, Korean Mini-Mental State Examination; NIHSS, National Institutes of Health Stroke Scale. 
(A)

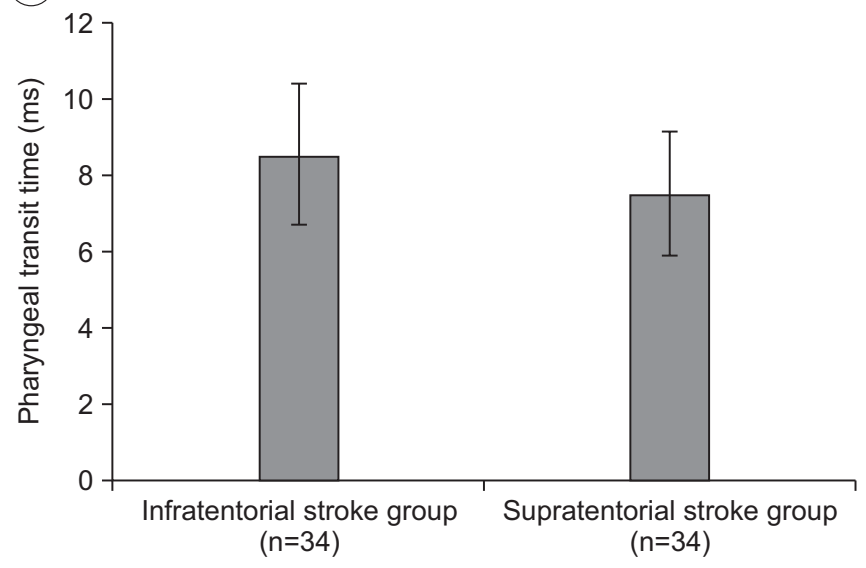

(C)

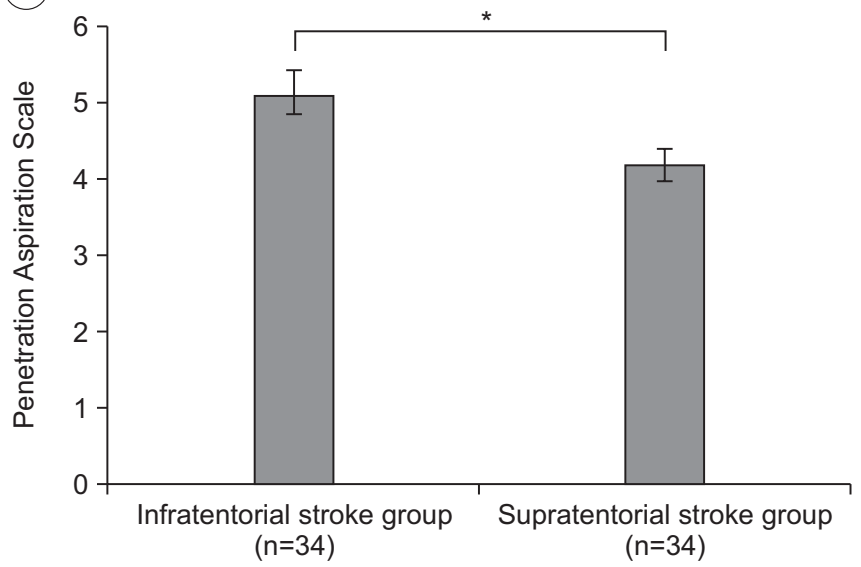

(B)

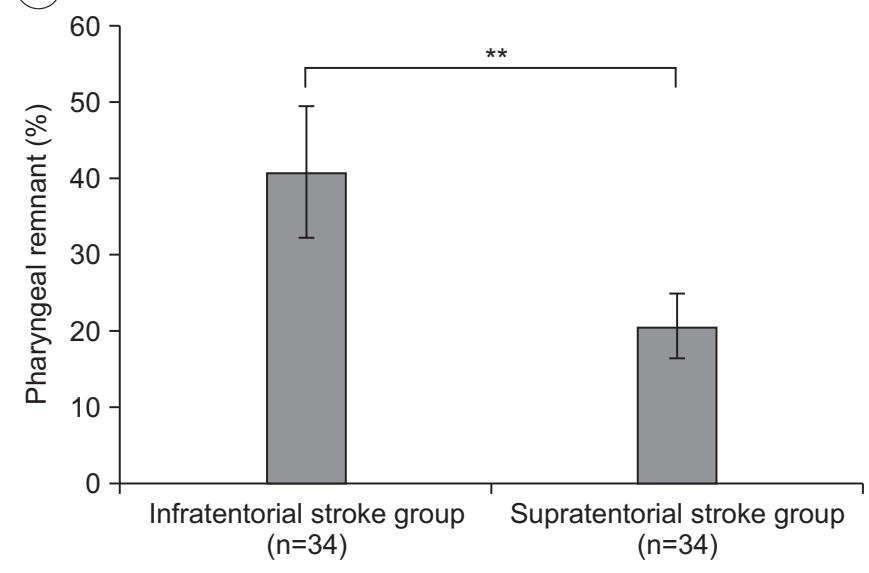

(D)

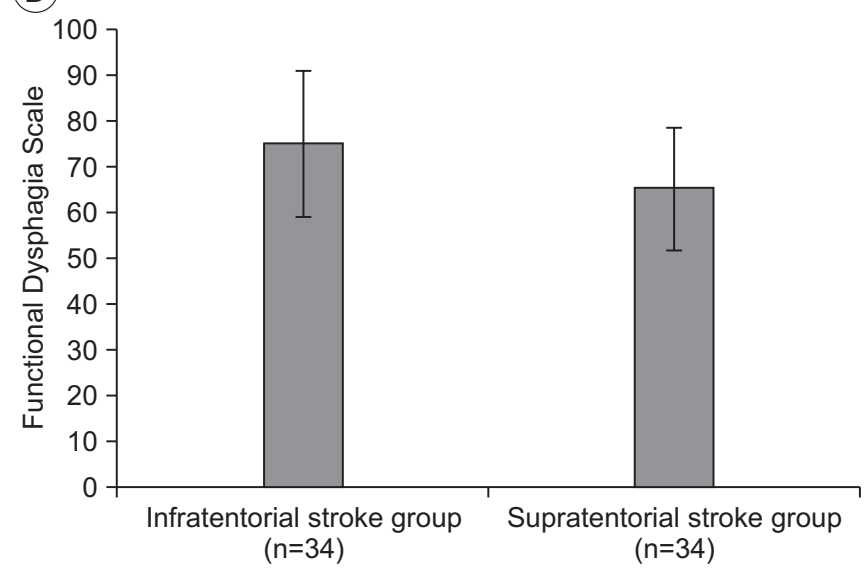

Fig. 1. Comparison of dysphagia between infratentorial and supratentorial stroke patients: (A) pharyngeal transit time, (B) pharyngeal remnant, (c) Penetration Aspiration Scale, and (D) Functional Dysphagia Scale. ${ }^{*} \mathrm{p}<0.05,{ }^{* *} \mathrm{p}<0.01$.

there is no statistical significance [16].

The PSD interferes with functional recovery. It may lead to death due to malnutrition or aspiration pneumonia [17]. This explains the importance of treatment for PSD. Patients with PSD may exhibit various symptoms such as leakage of food toward the oral cavity, reduced contraction of pharyngeal muscles, residue in the valleculae and the pyriform sinus, impairments in the mobility of the hyoid bone and the larynx, abnormal UES opening, and prolonged PTT [18]. Therefore, as shown in results of this study, infratentorial stroke patients can have an increased risk of aspiration pneumonia.

Another significant result from this study is that it suggests the following reasons for post-swallow pharyngeal remnant [4]: (1) insufficient pharyngeal propulsive force due to weak retraction of the tongue base and (2) insufficient opening of the UES. It has been shown that infraten- torial stroke patients have relatively insufficient opening of the UES [19]. However, there is a lack of a standardized or objective evaluation tool for evaluating UES opening. Currently, there are ongoing studies that evaluate the function of UES using manometry $[18,20]$. Once objective information is accumulated and tools are developed, evaluation of the UES opening will be possible. This in turn will help us assess the high risk of remnant and PAS score, similar to our current study. Furthermore, it may be possible to identify the association with development of cricopharyngeal dysfunction in infratentorial stroke patients that have relatively limited UES opening.

Compared to a recent study [21], patients in the present study had moderate to severe National Institutes of Health Stroke Scale (NIHSS). In the previous study [21], patients had relatively mild NIHSS. However, in the present study, severe stroke patients were recruited. Dyspha- 
gia is more likely to cause mortality in the severe stroke group. This can be a clinically meaningful result [22].

In this study, there were significant differences in pharyngeal remnant and PAS score, but there was no statistical difference in terms of PTT or FDS between infratentorial stroke patients and supratentorial stroke patients. Such findings might be attributed to several reasons. Since the pharyngeal remnant is a simple comparison of the amount of remnant, the probability of interference of other factors is not high. PAS score evaluates the risk of aspiration. It is associated with the amount of remnant [23]. Thus, the function of UES and protection of the vocal cords might be relatively decreased in the infratentorial group. Currently, although the evaluation of LES and gastric emptying is actively performed with tests like a manometry, UES evaluation is relatively neglected. If more studies on UES evaluation are performed in the future $[18,20]$, additional detailed evaluation related to the pathology of dysphagia (such as identifying whether the issue is with the relaxation time or the swallowing phase) and application of appropriate treatment methods will be possible.

FDS is a complex dysphagia scale that not only assesses the remnant amount, but also determines lip closure, bolus formation, and oral transit time [4]. In the present study, FDS showed no statistical significance. This is because FDS is an evaluation tool that can also affect the supratentorial group due to its complexity. Thus, such results are expected. Although comparison studies by FDS category and statistics were not described, lip closure was not adjusted in this study as it was influenced by the presence of facial palsy and patient's cognition. Bolus formation and oral transit time are indices that are highly associated with patient's cognition. Differences may occur due to cognition or factors others than difference in findings caused by location of the lesion. In addition, there was no significant difference of PTT between the two groups of this study. PTT measured in this study was set from the point of hyoid elevation to the point where the UES opened and the tail of the food bolus passed the UES [12]. Mechanisms involving pharyngeal wall contraction, swallowing, and opening and closing of the UES to smoothly allow passing of the food bolus are believed to be innervated by the glossopharyngeal nerve originating in the medulla and the vagus nerve [24]. Thus, in the infratentorial group including medullary lesions, PTT could be expected to be delayed. However, only 6 out of 34 patients in this group had medullary lesions, which might be a limited number compared to the control group in order to demonstrate reduced performance due to glossopharyngeal and vagus nerve damage. Furthermore, as reported by Perie et al. [25], cranial nerve damage associated with swallowing can vary according to the extent and severity of functional disorder. Two patients with damage solely to the vagus nerve can show both normal laryngeal sensation and initiation of swallowing. They can also have differences in the closing of the larynx or UES relaxation. Therefore, even within the infratentorial stroke group with medullary damage, the difference in developmental patterns may be a reason for an insignificant PTT result.

Malandraki et al. [26] have assessed the region of the brain that is activated during swallowing in the normal population using functional magnetic resonance imaging. They found that not only the cerebrum, but also the cerebellum and brainstem were activated. Malandraki et al. [26] have explained that because swallowing is a series of processes involving 55 muscles distributed across the mouth, larynx, pharynx and esophagus, nucleus and 6 cranial nerves in the pons and medulla, and two cervical nerve roots, dysphagia should be assessed as a declined comprehensive coordination rather than an issue in a specific area. However, as shown in this study, the severity of dysphagia was relatively higher in the infratentorial stroke group. Thus, more precise evaluation and customized treatment are necessary for this group.

This study has some limitations. First, ischemic and hemorrhagic stroke patients were analyzed together. In case of lesion specific ischemic stroke, patterns of dysphagia may differ from that of hemorrhagic stroke. For hemorrhagic stroke, the effect on other regions due to the mass effect of ICH was not evaluated. Therefore, if future analyses could be done on the possible midline shift or other findings of increased intracranial pressure by reviewing the brain image, another important point could be made. Second, we enrolled a small number of patients in the current analysis. Third, we only evaluated patients who were hospitalized at a single tertiary medical institution. Therefore, we could not completely rule out the possibility of selection bias.

In conclusion, the severity of dysphagia in the infratentorial stroke group is more severe than that in the supra- 
tentorial stroke group. This may be due to larger relative association with the swallowing center. Therefore, a different therapeutic approach from that of dysphagia in the infratentorial group may be necessary. Appropriate treatment could be subsequently applied to the stroke segment or lesion. More precise analysis of dysphagia is needed in the future.

\section{CONFLICT OF INTEREST}

No potential conflict of interest relevant to this article was reported.

\section{AUTHOR CONTRIBUTION}

Conceptualization: Kim YK, Lee KY. Methodology: Kim YK, Lee KY. Formal analysis: Lee KY, Cha JH. Funding acquisition: not applicable. Project administration: Kim YK. Visualization: Kim YK, Lee KY. Writing - original draft: Lee KY. Writing - review and editing: Lee KY. Approval of final manuscript: all authors.

\section{REFERENCES}

1. Arnold M, Liesirova K, Broeg-Morvay A, Meisterernst J, Schlager M, Mono ML, et al. Dysphagia in acute stroke: incidence, burden and impact on clinical outcome. PLoS One 2016;11:e0148424.

2. Gonzalez-Fernandez M, Ottenstein L, Atanelov L, Christian AB. Dysphagia after stroke: an overview. Curr Phys Med Rehabil Rep 2013;1:187-96.

3. Kim BR, Moon WJ, Kim H, Jung E, Lee J. Association of dysphagia with supratentorial lesions in patients with middle cerebral artery stroke. Ann Rehabil Med 2016; 40:637-46.

4. Han TR, Paik NJ, Park JW. Quantifying swallowing function after stroke: a functional dysphagia scale based on videofluoroscopic studies. Arch Phys Med Rehabil 2001;82:677-82.

5. Kameda W, Kawanami T, Kurita K, Daimon M, Kayama T, Hosoya $\mathrm{T}$, et al. Lateral and medial medullary infarction: a comparative analysis of 214 patients. Stroke 2004;35:694-9.

6. Marik PE, Kaplan D. Aspiration pneumonia and dysphagia in the elderly. Chest 2003;124:328-36.

7. Sharma JC, Fletcher S, Vassallo M, Ross I. What influ- ences outcome of stroke: pyrexia or dysphagia? Int J Clin Pract 2001;55:17-20.

8. Suntrup S, Kemmling A, Warnecke T, Hamacher C, Oelenberg $\mathrm{S}$, Niederstadt $\mathrm{T}$, et al. The impact of lesion location on dysphagia incidence, pattern and complications in acute stroke. Part 1: dysphagia incidence, severity and aspiration. Eur J Neurol 2015;22:832-8.

9. Meng NH, Wang TG, Lien IN. Dysphagia in patients with brainstem stroke: incidence and outcome. Am J Phys Med Rehabil 2000;79:170-5.

10. Horner J, Buoyer FG, Alberts MJ, Helms MJ. Dysphagia following brain-stem stroke: clinical correlates and outcome. Arch Neurol 1991;48:1170-3.

11. Kim YK, Choi SS, Choi JH, Yoon JG. Effectiveness of rehabilitative balloon swallowing treatment on upper esophageal sphincter relaxation and pharyngeal motility for neurogenic dysphagia. Ann Rehabil Med 2015;39:524-34.

12. Kim JC, Kim JS, Jung JH, Kim YK. The effect of balloon dilatation through video-fluoroscopic swallowing study (VFSS) in stroke patients with cricopharyngeal dysfunction. J Korean Acad Rehabil Med 2011;35:23-6.

13. McHorney CA, Robbins J, Lomax K, Rosenbek JC, Chignell K, Kramer AE, et al. The SWAL-QOL and SWAL-CARE outcomes tool for oropharyngeal dysphagia in adults. III. Documentation of reliability and validity. Dysphagia 2002;17:97-114.

14. Martin-Harris B, Brodsky MB, Michel Y, Ford CL, Walters B, Heffner J. Breathing and swallowing dynamics across the adult lifespan. Arch Otolaryngol Head Neck Surg 2005;131:762-70.

15. Singh S, Hamdy S. Dysphagia in stroke patients. Postgrad Med J 2006;82:383-91.

16. Sue Eisenstadt E. Dysphagia and aspiration pneumonia in older adults. J Am Acad Nurse Pract 2010;22:1722.

17. Paik NJ, Kim IS, Kim JH, Oh BM, Han TR. Clinical validity of the functional dysphagia scale based on videofluoroscopic swallowing study. J Korean Acad Rehabil Med 2005;29:43-9.

18. Park CH, Kim DK, Lee YT, Yi Y, Lee JS, Kim K, et al. Quantitative analysis of swallowing function between dysphagia patients and healthy subjects using highresolution manometry. Ann Rehabil Med 2017;41:77685.

19. Kim Y, Park T, Oommen E, McCullough G. Upper 
esophageal sphincter opening during swallow in stroke survivors. Am J Phys Med Rehabil 2015;94:7349.

20. Lee H, Chung H, Lee TH, Hong KS, Youn YH, Park $\mathrm{JH}$, et al. Therapeutic outcome of achalasia based on high-resolution manometry: a Korean multicenter study. Am J Ther 2017 Sep 11 [Epub]. http://doi.org/ 10.1097/MJT.0000000000000677.

21. Daniels SK, Pathak S, Mukhi SV, Stach CB, Morgan $\mathrm{RO}$, Anderson JA. The relationship between lesion localization and dysphagia in acute stroke. Dysphagia 2017;32:777-84.

22. Yuan MZ, Li F, Fang Q, Wang W, Peng JJ, Qin DY, et al. Research on the cause of death for severe stroke patients. J Clin Nurs 2018;27:450-60.

23. Steele CM, Grace-Martin K. Reflections on clinical and statistical use of the penetration-aspiration scale. Dysphagia 2017;32:601-16.

24. Kiyokawa J, Yamaguchi K, Okada R, Maehara T, Akita $\mathrm{K}$. Origin, course and distribution of the nerves to the posterosuperior wall of the external acoustic meatus. Anat Sci Int 2014;89:238-45.

25. Perie S, Coiffier L, Laccourreye L, Hazebroucq V, Chaussade S, St Guily JL. Swallowing disorders in paralysis of the lower cranial nerves: a functional analysis. Ann Otol Rhinol Laryngol 1999;108:606-11.

26. Malandraki GA, Sutton BP, Perlman AL, Karampinos DC, Conway C. Neural activation of swallowing and swallowing-related tasks in healthy young adults: an attempt to separate the components of deglutition. Hum Brain Mapp 2009;30:3209-26. 